\title{
Detailed Spectral Modeling of a 3-D Pulsating Reverse Detonation Model: Too Much Nickel
}

\author{
E. Baron, ${ }^{1,2}$ David J. Jeffery, ${ }^{1,3}$ David Branch, ${ }^{1}$ Eduardo Bravo, ${ }^{4,5}$ Domingo García-Senz, ${ }^{4,5}$ \\ and Peter H. Hauschildt ${ }^{6}$
}

\begin{abstract}
We calculate detailed NLTE synthetic spectra of a Pulsating Reverse Detonation (PRD) model, a novel explosion mechanism for Type Ia supernovae. While the hydro models are calculated in 3-D, the spectra use an angle averaged hydro model and thus some of the 3-D details are lost, but the overall average should be a good representation of the average observed spectra. We study the model at 3 epochs: maximum light, seven days prior to maximum light, and 5 days after maximum light. At maximum the defining Si II feature is prominent, but there is also a prominent C II feature, not usually observed in normal SNe Ia near maximum. We compare to the early spectrum of SN 2006D which did show a prominent C II feature, but the fit to the observations is not compelling. Finally we compare to the post-maximum UV+optical spectrum of SN 1992A. With the broad spectral coverage it is clear that the iron-peak elements on the outside of the model push too much flux to the red and thus the particular PRD realizations studied would be intrinsically far redder than observed SNe Ia. We briefly discuss variations that could improve future PRD models.
\end{abstract}

Subject headings: stars: atmospheres — supernovae: SN 1992A, SN 1994D, SN $2006 \mathrm{D}$

\footnotetext{
${ }^{1}$ Homer L. Dodge Department of Physics and Astronomy, University of Oklahoma, 440 West Brooks, Rm. 100, Norman, OK 73019-2061; USA, baron,branch,jeffery@nhn.ou.edu

${ }^{2}$ Computational Research Division, Lawrence Berkeley National Laboratory, MS 50F-1650, 1 Cyclotron Rd, Berkeley, CA 94720 USA

${ }^{3}$ Department of Physics, University of Idaho, PO Box 440903, Moscow, ID 83844-0903

${ }^{4}$ Departament Física i Enginyeria Nuclear, Universitat Politècnica de Catalunya, Diagonal 647, 08028 Barcelona, Spain; eduardo.bravo,domingo.garcia@upc.edu

${ }^{5}$ Institut d'Estudis Espacials de Catalunya, Barcelona, Spain

${ }^{6}$ Hamburger Sternwarte, Gojenbergsweg 112, 21029 Hamburg, Germany; yeti@hs.uni-hamburg.de
} 


\section{Introduction}

Understanding the nature of the explosion mechanism of Type Ia supernovae (SNe Ia) is important for the use of SNe Ia as precision cosmological probes as well as for stellar evolution and nucleosynthesis. Determining something about the nature of the explosion will almost certainly shed light on the physical explanation of the empirical light curve shape-luminosity relationship (Phillips 1993; Riess et al. 1995, 1996; Phillips et al. 1999; Goldhaber et al. 2001; Kasen \& Woosley 2007) as well as further physical parameters associated with the observed diversity in the SN Ia population. Understanding the physical nature of the diversity is crucial in being able to estimate systematic errors that may arise in comparing the characteristics of the nearby sample with the cosmological one.

While there is widespread agreement that the $\mathrm{SN}$ Ia progenitor consists of a $\mathrm{C}+\mathrm{O}$ white dwarf that accretes material from a companion and ignites the $\mathrm{C}+\mathrm{O}$ fuel when the Chandrasekhar mass is reached, the exact mechanism of explosion is not known. The explosion could take the form of a super-sonic detonation (shock-wave), or a sub-sonic deflagration. If a unexpanded Chandrasekhar mass WD detonates, the resulting products would be almost entirely ${ }^{56} \mathrm{Ni}$ and $\alpha$-particles (Arnett et al. 1971), contrary to the signature intermediate mass elements of silicon, sulfur, and calcium that define the SNe Ia class. For many years the standard SN Ia model has been the 1-D, parameterized, deflagration model W7 (Nomoto et al. 1984). In that model the speed of the flame was adjusted in order to reproduce the approximately correct amount of ${ }^{56} \mathrm{Ni}$, as well as intermediate mass elements. Nevertheless, the central regions of the model undergo significant electron capture and far too much neutron-rich material is produced, polluting the interstellar medium. Brachwitz et al. (2000) showed that with pf-shell model calculations the electron capture rates are reduced over those of standard sd-shell rates of Fuller et al. (1980; 1982b; 1982a; 1985), we are unaware of hydrodynamical calculations that show that the problem is completely solved. In order to avoid some of the problems of W7, the delayed-detonation model has gained significant favor (Gamezo et al. 2005, 2004; Khokhlov 1993, 1991a, b). In this scenario, the ignition begins as a deflagration, allowing the star to pre-expand, before the deflagration transforms into a detonation at lower densities, which produces both ${ }^{56} \mathrm{Ni}$ and intermediate mass elements. While this process is known to occur in confined terrestrial situations, it is not known whether it can take place in the unconfined white dwarf.

The last decade has seen the beginning of full 3-D hydro calculations (Reinecke et al. 2002a.,b.c;; Hillebrandt et al. 2000; Reinecke et al. 1999a,b; Gamezo et al. 2003, 2004, 2005; García-Senz \& Bravo 2005; Bravo \& García-Senz 2003; García-Senz \& Bravo 2003; Garcia-Senz et al. 1999; Röpke et al. 2006; Röpke et al. 2006; Röpke \& Hillebrandt 2005a,b; Röpke 2005). Much of the work has focused on pure deflagration models as well as developing the necessary 
sub-grid techniques to handle the essentially infinite dynamic range (due to turbulence) or studying the effects of differing numbers of ignition points. The initial ignition conditions may well be unknowable since about one hundred years before ignition the white dwarf becomes turbulent (Röpke et al. 2007). Recently, there has been general agreement that pure deflagration models leave behind significant amounts of unburnt carbon and oxygen and that the kinetic energy of the explosion is too low to reproduce the observed spectra of normal SNe Ia Gamezo et al. 2004; Mazzali et al. 2007). The alternative is a delayed detonation (Khokhlov 1993, 1991a. b. . . ; Gamezo et al. 2005; Mazzali et al. 2007) where the deflagration accelerates into a detonation or some other process that turns the deflagration into a detonation. Two alternatives that act in a similar manner to the delayed-detonation scenario are the "Gravitationally Confined Detonation" (Plewa et al. 2004; Jordan et al. 2007) and the "Pulsating Reverse Detonation" (Bravo \& García-Senz 2006).

We present detailed NLTE synthetic spectral calculations of an angle averaged version of PRD5.5 a pulsating-reverse-detonation calculation (E. Bravo \& D. García-Senz, in preparation). The detailed chemical composition was determined from the hydro model with a post-processing code that included a maximum of 725 isotopes. The evolution of the electron fraction $Y_{e}$ was followed along with the hydrodynamic calculation with the aid of tables of electron capture rates in NSE matter. The procedure was identical to that used in Garcia-Senz et al. (1999).

\section{Calculations}

The calculations were performed using the multi-purpose stellar atmospheres program PHOENIX version 15 Hauschildt \& Baron 1999; Baron \& Hauschildt 1998; Hauschildt et al. 1997a,b, 1996). Version 15 incorporates many changes over previous versions used for supernova modeling (Baron et al. 2007, 2006) including many more species in the equation of state (83 versus 40), twice as many atomic lines, and many more species treated in full NLTE. PHOENIX solves the radiative transfer equation along characteristic rays in spherical symmetry including all special relativistic effects. The non-LTE (NLTE) rate equations for many ionization states are solved including the effects of ionization due to non-thermal electrons from the $\gamma$-rays produced by the radiative decay of ${ }^{56} \mathrm{Ni}$, which is produced in the supernova explosion. The atoms and ions calculated in NLTE are: H I, He I-II, C I-III, O I-III, Ne I, Na I-II, Mg I-III, Si I-III, S I-III, Ca II, Ti II, Fe I-III, Ni I-III, and Co I-III. These are all the elements whose features make important contributions to the observed spectral features in SNe Ia.

Each model atom includes primary NLTE transitions, which are used to calculate the 
level populations and opacity, and weaker secondary LTE transitions which are included in the opacity and implicitly affect the rate equations via their effect on the solution to the transport equation (Hauschildt \& Baron 1999). In addition to the NLTE transitions, all other LTE line opacities for atomic species not treated in NLTE are treated with the equivalent two-level atom source function, using a thermalization parameter, $\alpha=0.10$ (Baron et al. 1996). The atmospheres are iterated to energy balance in the co-moving frame; while we neglect the explicit effects of time dependence in the radiation transport equation, we do implicitly include these effects, via explicitly including the rate of gamma-ray deposition in the generalized equation of radiative equilibrium and in the rate equations for the NLTE populations.

The outer boundary condition is the total bolometric luminosity in the observer's frame. The inner boundary condition is that the flux at the innermost zone $\left(v=1500 \mathrm{~km} \mathrm{~s}^{-1}\right)$ is given by the diffusion equation. We treat the $\gamma$-ray deposition by solving the full radiative transfer for a grey opacity. Detailed fitting of the observed spectra determines all the parameters.

PHOENIX has been well tested on SNe Ia (Nugent et al. 1995a, b, 1997; Lentz et al. 2001; Baron et al. 2006) and particularly on SN 1994D (Lentz et al. 2001; Baron et al. 2006). PHOENIX is subjected to rigorous regression testing with each new version.

\section{The Model}

In the Pulsating Reverse Detonation model (Bravo \& García-Senz 2006) the deflagration starts in a number of rising plumes that are already somewhat close to the surface. These plumes burn without much expansion of the white dwarf and hence burn to iron peak elements. The energy released by this initial deflagration is not enough to unbind the star and the outer parts fall back producing an accretion shock. The unburnt core settles back into equilibrium and the accretion shock detonates the outer parts which are at low enough density that they burn to intermediate mass elements. Thus the composition structure in this model is not completely stratified and the outer layers are polluted by iron and radioactive nickel. The precise amount of polluting Fe-group elements is sensitive to the outcome of the deflagrative phase of the explosion, more efficient subsonic burning reduces the amount of stable Fe-group pollution. The mass fraction of radioactive material close to the surface of the ejecta depends as well on the amount of electron captures that, in turn, are a sensitive function of the central density at ignition.

We present results from model PRD5.5 (E. Bravo \& D. García-Senz, in preparation). 
The model started from five sparks, incinerating $0.14 \mathrm{M}_{\odot}$ during the initial subsonic combustion phase, and later on a detonation processed most of the remaining carbon and oxygen. Finally, the explosion produced $1.3 \times 10^{51}$ ergs of kinetic energy, and ejected $0.86 \mathrm{M}_{\odot}$ of ${ }^{56} \mathrm{Ni}, 0.15 \mathrm{M}_{\odot}$ of intermediate-mass elements, and $0.18 \mathrm{M}_{\odot}$ of carbon and oxygen. In model PRD5.5 the ejected mass of stable iron and nickel was $0.1 \mathrm{M}_{\odot}$, most of which was moving at velocities in excess of $10,000 \mathrm{~km} \mathrm{~s}^{-1}$. The final averaged distribution of elements in velocity space is quite similar to that of model PRD6 (see Fig. 3 in Bravo \& García-Senz 2006).

The 3-D composition of model PRD5.5 is quite clumpy. The angle averaged version is rather uniform in the outer layers above $\sim 8000 \mathrm{~km} \mathrm{~s}^{-1}$, where the supernova spectrum mostly forms before a few days past maximum light (Fig. 1). While the outer layers are dominated by ${ }^{56} \mathrm{Ni}$, there is significant silicon, sulfur, and oxygen as required for SN Ia spectra. It is a quantitative question that only a code such as PHOENIX can determine whether or not the outer ${ }^{56} \mathrm{Ni}$ conflicts with observations. There is also significant carbon in the outer layers and $\mathrm{C}+\mathrm{O}$ dominates the innermost region. Synthetic spectrum modeling by Kozma et al. (2005) of the deflagration model c3_3d_256_10s (Röpke 2005) found that abundant $\mathrm{C}+\mathrm{O}$ matter in the interior was inconsistent with late-time nebular spectra. Model c3_3d_256_10s had more interior $\mathrm{C}+\mathrm{O}$ than PRD5.5, therefore future nebular modeling of PRD5.5 is also important.

\section{Results}

We first examine the maximum light spectrum of SN 1994D, which we have used in previous papers to study the detailed spectral behavior since it is nearby and extremely well-observed (Branch et al. 2005; Lentz et al. 2001). Figure 2 shows the results of our bestfit model (we use the outer boundary condition of the total bolometric luminosity in the observer's frame to find the best fit). The overall fit is not all that bad, the feature at $6150 \AA$ attributed to Si II $\lambda 6355$ is reasonably well fit, however the "W" feature attributed to S II is totally washed out. Additionally just to the red of the 6150 feature is another feature almost certainly due to C II $\lambda 6580$. This feature has in fact been observed in early SNe Ia spectra (Branch et al. 2003; Thomas et al. 2007). While the $6150 \AA$ feature is the usual defining feature for SN Ia, the S II "W" is even a more reliable diagnostic. SNe Ib/c spectra do show features that have been identified as Si II, but only SNe Ia show the S II feature. Since silicon and sulfur are made nearly simultaneously one might expect the lines to appear together, however the Si II line is much stronger than the S II lines, thus it requires more sulfur to obtain the S II "W" feature. Empirically S II seems to be the best classifier

of SNe Ia. Even the extremely odd SN Ia 2002ic which shows strong narrow Balmer lines 


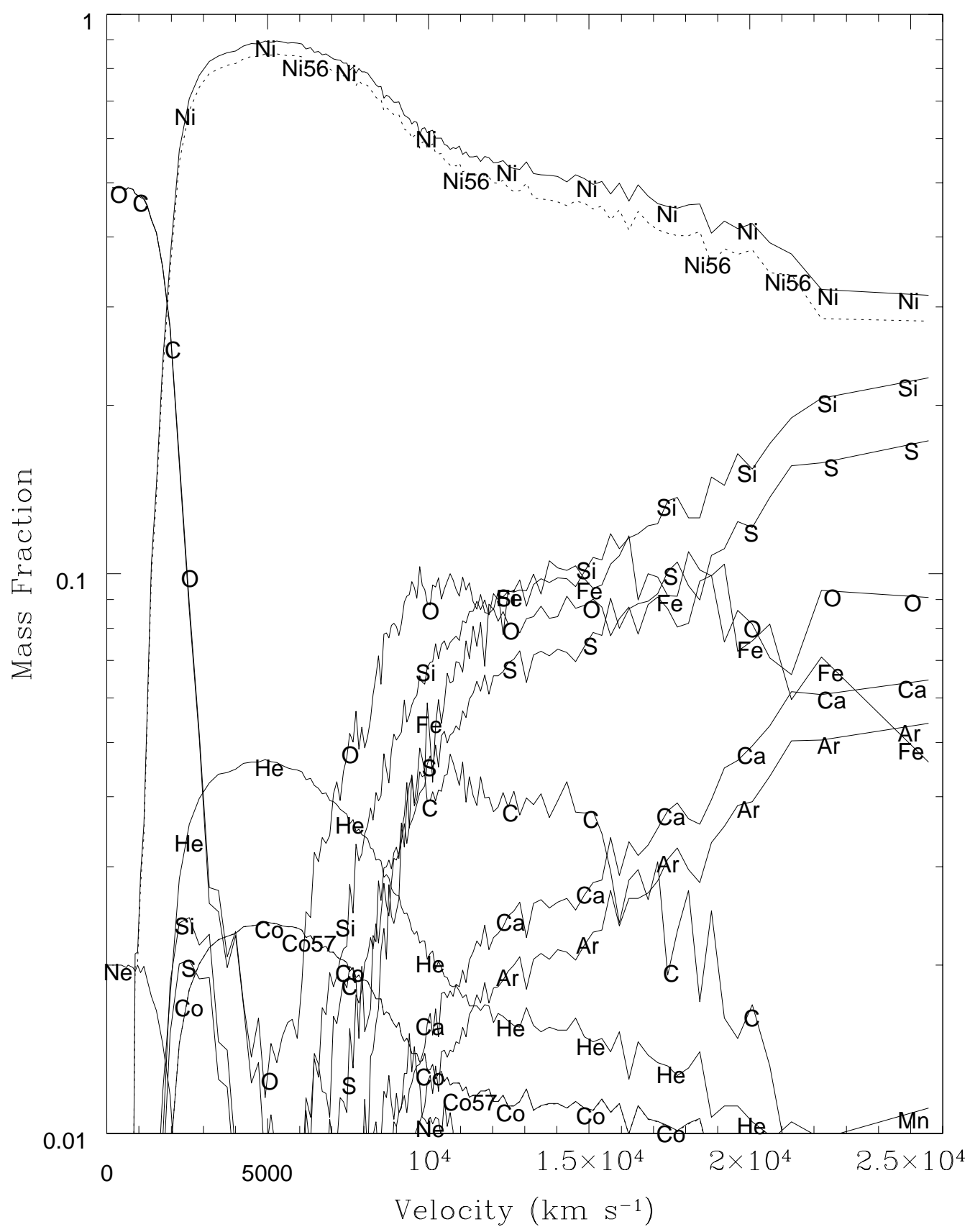

Fig. 1. - The angle averaged composition of the PRD5.5 at time zero after the explosion: i.e., before significant radioactive decay has occur. Elements are denoted by solid curves; radioactive nuclides by dotted curves. 
in emission shows solid evidence for the S II "W" (Hamuy et al. 2003). If S II is used as the defining feature of SN Ia, then the synthetic spectrum of Fig. 2 is not that of a SN Ia. Since the angle averaging will automatically lead to some washing out of the flux spectrum we should be careful not to draw too strong conclusions about the absence of S II lines, but their complete absence in the presence of $\mathrm{Si}$ II is interesting. While the relative amount of silicon and sulfur in the model is quite similar S III is less abundant than Si III only by a factor of two or more everywhere in the outer atmosphere, thus it is unclear why there is no evidence for S II in the synthetic spectra (the lines of the ionization stage just below the most abundant one tend to be strongest, since there is then energy to excite the levels above the ground state). We speculate that the complete absence of evidence for S II in the synthetic spectrum is a peculiarity of the particular NLTE state realized in the outer ejecta of PRD5.5. If so (and only a very detailed decomposition of the spectrum formation in future work can tell), only a PHOENIX level calculation could reveal this peculiarity inherent in PRD5.5.

Figure 3 shows the best fit spectrum compared to SN 2006D seven days prior to $B$ maximum, where the C II $\lambda 6580$ line is prominent in the observed spectrum. At this earlier time the emission portion of the carbon line in the synthetic spectrum is evident but the absorption trough is not blue enough and does not have the right shape. The triangular shape in the observed spectrum is almost certainly due to line blending. S II is again missing in the synthetic spectrum. The outermost compositions used in the spectral modelling are somewhat uncertain due to lower resolution in the outer parts of the spectral grid and to the need to interpolate from the gridless hydro models (the grid needed for good resolution in synthetic spectra is different from that needed in hydro). This effect, coupled with the smearing created by the angle averaging procedure would lead us not to expect perfect agreement with the observed lineshapes.

One of the key features of the pulsating-reverse-detonation model is that it creates a composition inversion. That is, the initial deflagration burns at high density and thus tends to make iron-peak elements on the outside at high velocity. High velocity features have been detected in supernova spectra at early times, but they tend to be either unburnt material such as carbon, or intermediate mass elements like calcium. Branch et al. (2006) used high velocity Fe II to fit near maximum light supernova spectra, using the simple parameterized code SYNOW. However, the optical depths used were low, and thus the material could be primordial. It is impossible to use SYNOW to directly determine abundances. Figure 4 shows the combined HST and optical spectrum of SN 1992A five days past maximum light (Kirshner et al. 1993). Plotted on this figure are five different choices for the outer boundary condition, the total luminosity in the observer's frame. The luminosity varies from the lowest value to the highest by a factor of 13 . The logarithmic bolometric luminosity $\left(\mathrm{ergs} \mathrm{s}^{-1}\right)$ is: 


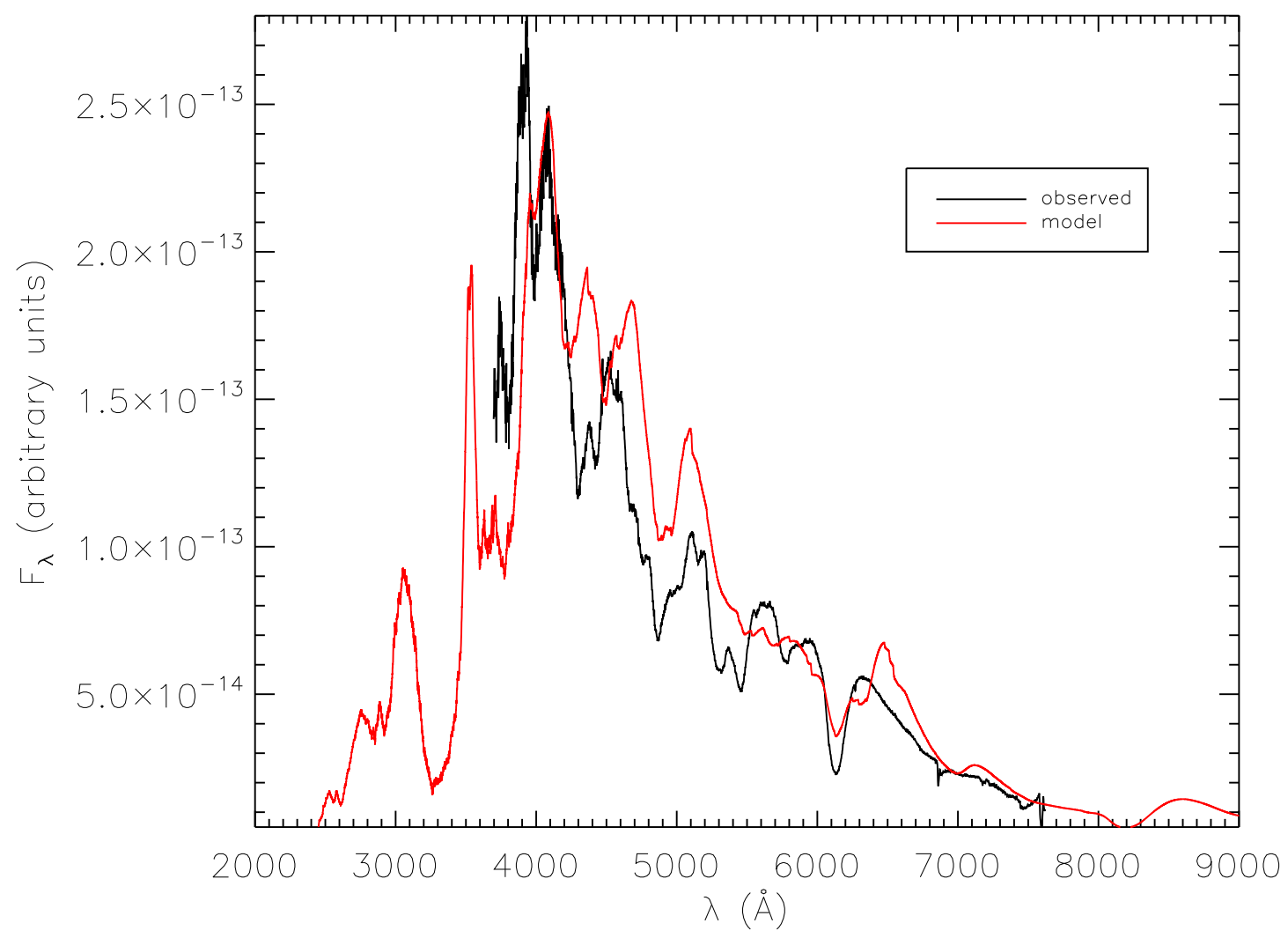

Fig. 2.- The synthetic spectra of a full NLTE model of PRD5.5, 20 days after explosion, is compared to the observed spectrum of SN 1994D on March 21, 1994 (the time of B maximum). The observed spectrum has been corrected for redshift assuming a velocity of $448 \mathrm{~km} \mathrm{~s}^{-1}$ and a reddening of $E(B-V)=0.06$. 


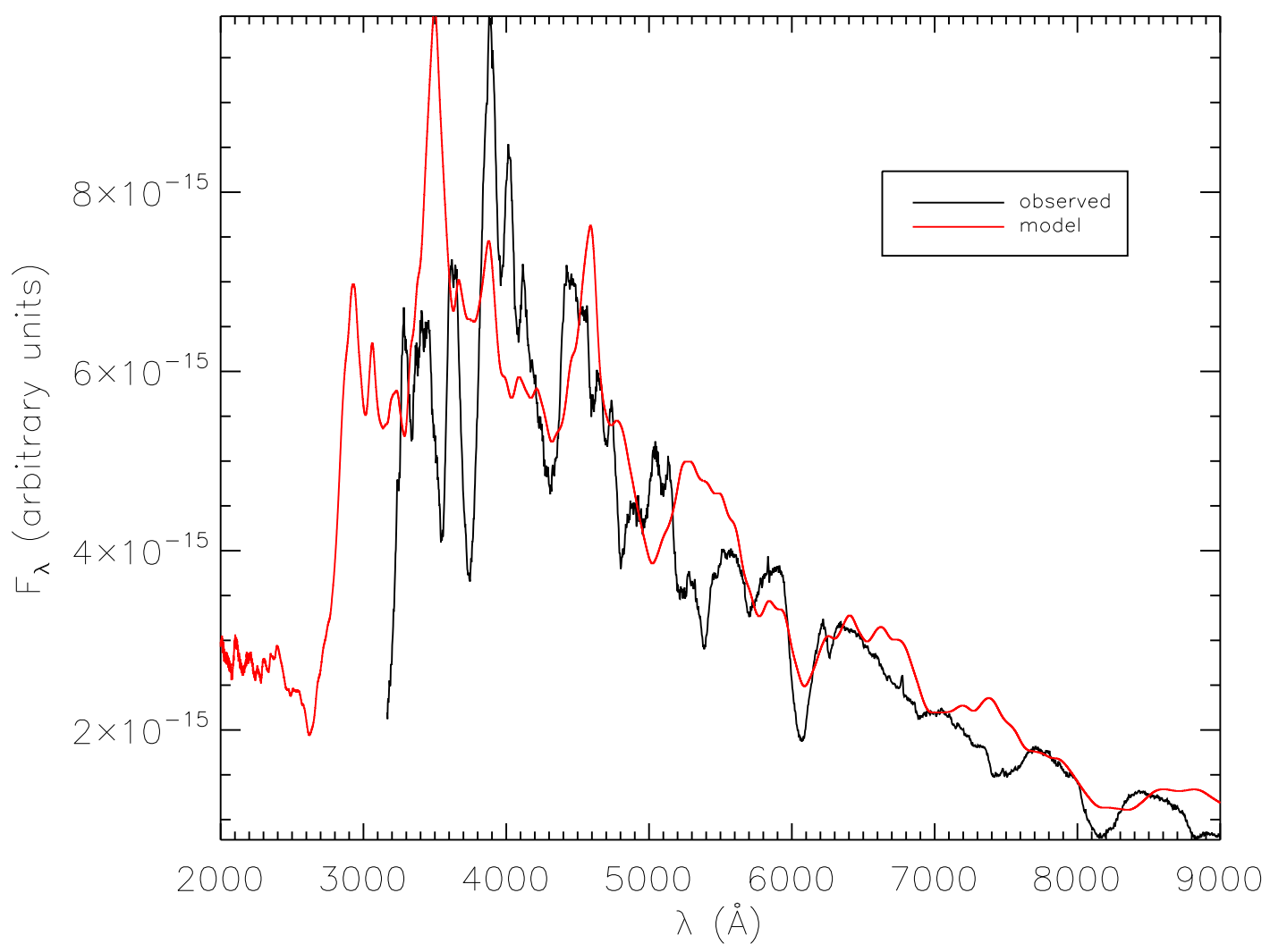

Fig. 3.- The synthetic spectra of a full NLTE model of PRD5.5, 13 days after explosion, is compared to the observed spectrum of SN 2006D on 7 days prior to B maximum. The observed spectrum has been corrected for redshift $z=0.00853$. No reddening correction was made. 
$\mathrm{L} 1=-17.02, \mathrm{~L} 2=-18.58, \mathrm{~L} 3=-19.07, \mathrm{~L} 4=-19.31, \mathrm{~L} 5=-19.81$. Clearly no value chosen for the luminosity can reproduce the observed colors across the UV+optical spectrum. This is almost certainly due to the large amount of iron-peak elements in the outer parts of the model. These elements have such strong line blanketing that they always push much of the flux to the red and thus the red colors are too red for all reasonable luminosities.

\section{Conclusions}

The pulsating-reverse-detonation model is a novel mechanism that combines the advantages of the delayed detonation mechanism (higher explosion energy, low amounts of unburnt fuel remaining) without requiring a deflagration to detonation transition in an unconfined medium. However, the mechanism still has to be explored further and different variations will change the total mass in the initial deflagration. We have examined just one particular realization here and we have also studied only the angle averaged flux spectra calculated in spherical symmetry, thus losing some of the important 3-D effects of the model. We note that we have also studied the spectra of PRD6.0. PRD6.0 burned carbon more efficiently during the deflagration phase, thus the mass fraction of stable iron and nickel in the outer layers of that model is nearly one order of magnitude lower than in PRD5.5. However, the mass fraction of ${ }^{56} \mathrm{Ni}$ in the outermost layer is only slightly lower $(\sim 15 \%)$ in PRD6.0. The overall differences in the synthetic spectra were not significant. This suggests that the composition inversion inherent in the model will lead to supernova spectra and light curves that are extremely red, which has not been observed. A way out of this would be if there were some pre-expansion during the initial deflagration phase so that the burning is to intermediate mass elements rather than all the way to the iron-peak. The pre-expansion is dependent on details of the initial model. For instance, if a shallow enough thermal gradient were present in the bubbles, the nuclear burning would propagate initially with a shockless supersonic phase velocity that would lead to pre-expansion before the combustion settled in a subsonic flame regime. Another possibility is that the explosion takes place in the core of a rotating white dwarf. Since for the same central density, fast rotators are more prone to synthesize more intermediate-mass elements and less iron than static initial models. The determination of the ignition configuration of the white dwarf is one of the key problems in the physics of SNIa during the explosion phase. Thus, we reiterate that the problem found in the models studied here do not rule out the PRD scenario, just the particular realizations that we have studied. Clearly there is more work to be done in studying this particular explosion mechanism. Our work also shows the power of quantitative spectroscopy in validating hydrodynamical explosion models. 


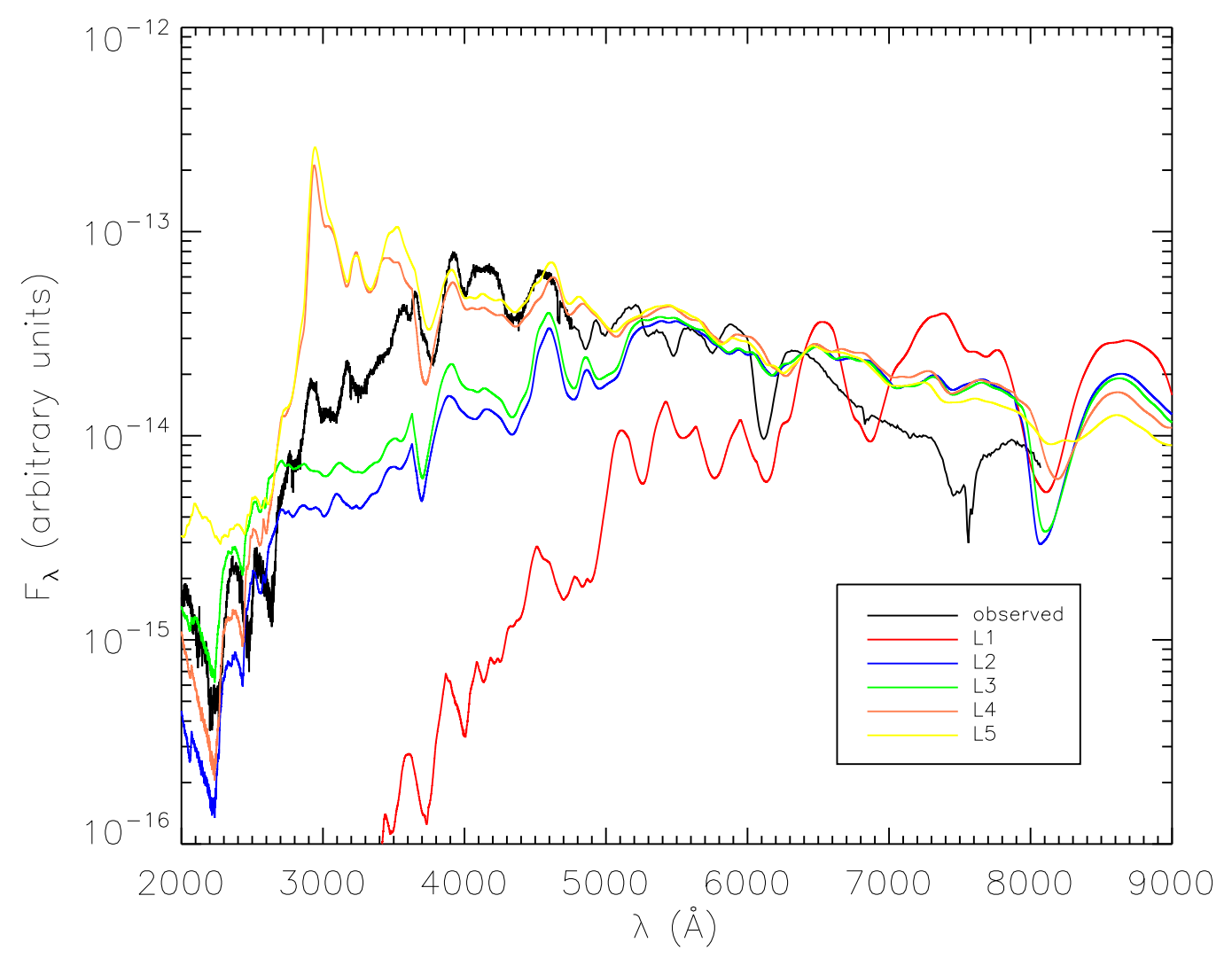

Fig. 4.- The synthetic spectra of a full NLTE model of PRD5.5, 25 days after explosion, are compared to the observed spectrum of SN 1992A observed five days past B maximum. The indicated lines show synthetic spectra of models with increasing total bolometric luminosity in the observer's frame (L1-L5). The observed spectrum has been corrected for redshift assuming a velocity of $1845 \mathrm{~km} \mathrm{~s}^{-1}$. No reddening correction was made. While the optical spectra can be somewhat fit, no variation in the luminosity can fit the entire wavelength range from the UV to the optical. 
We thank the referee for a constructive report that improved the presentation. This work was supported in part by NASA grant NNG04GB36G, US DOE Grant DE-FG0207ER41517, and NSF grants AST-0506028 and AST-0707704. E. Bravo and D.G.-S. have been supported by the MEC grants AYA2005-08013-C03, AYA2004-06290-C02, by the European Union FEDER funds and by the Generalitat de Catalunya. E. Baron acknowledges support from the US Department of Energy Scientific Discovery through Advanced Computing program under contract DE-FG02-06ER06-04. This research used resources of the National Energy Research Scientific Computing Center (NERSC), which is supported by the Office of Science of the U.S. Department of Energy under Contract No. DE-AC03-76SF00098;

and the Höchstleistungs Rechenzentrum Nord (HLRN). We thank all these institutions for a generous allocation of computer time.

\section{REFERENCES}

Arnett, W. D., Truran, J. W., \& Woosley, S. E. 1971, ApJ, 165, 87

Baron, E., Bongard, S., Branch, D., \& Hauschildt, P. 2006, ApJ, 645, 480

Baron, E., Branch, D., \& Hauschildt, P. H. 2007, ApJ, 662, 1148

Baron, E. \& Hauschildt, P. H. 1998, ApJ, 495, 370

Baron, E., Hauschildt, P. H., Nugent, P., \& Branch, D. 1996, MNRAS, 283, 297

Brachwitz, F., Dean, D., Hix, W. R., Iwamoto, K., Langanke, K., Martinez-Pinedo, G., Nomoto, K., Strayer, M., Thielemann, F.-K., \& Umeda, H. 2000, ApJ, 536, 934

Branch, D., Baron, E., Hall, N., Melakayil, M., \& Parrent, J. 2005, PASP, 117, 545

Branch, D., Dang, L. C., Hall, N., Ketchum, W., Melakayil, M., Parrent, J., Troxel, M. A., Casebeer, D., Jeffery, D. J., \& Baron, E. 2006, PASP, 118, 560

Branch, D., Garnavich, P., Matheson, T., Baron, E., Thomas, R. C., Hatano, K., Challis, P., Jha, S., \& Kirshner, R. P. 2003, ApJ, 128, 1489

Bravo, E. \& García-Senz, D. 2003, in From Twilight to Highlight: The Physics of Supernovae, ed. W. Hillebrandt \& B. Leibundgut, ESO Astrophysics Symposia (Berlin: Springer), 165

Bravo, E. \& García-Senz, D. 2006, ApJ, 642, L157

Fuller, G. M., Fowler, W. A., \& Newman, M. J. 1980, ApJS, 42, 447 
-. 1982a, ApJ, 252, 715

—. 1982b, ApJS, 48, 279

—. 1985, ApJ, 293, 1

Gamezo, V., Khokhlov, A., \& Oran, E. 2004, Phys. Rev. Lett., 92, 211102

—. 2005, ApJ, 623, 337

Gamezo, V. N., Khokhlov, A. M., Oran, E. S., Ctchelkanova, A. Y., \& Rosenberg, R. O. 2003, Science, 299, 77

García-Senz, D. \& Bravo, E. 2003, in From Twilight to Highlight: The Physics of Supernovae, ed. W. Hillebrandt \& B. Leibundgut, ESO Astrophysics Symposia (Berlin: Springer), 158

García-Senz, D. \& Bravo, E. 2005, A\&A, 430, 585

Garcia-Senz, D., Bravo, E., \& Woosley, S. E. 1999, A\&A, 349, 177

Goldhaber, G. et al. 2001, ApJ, 558, 359

Hamuy, M., Phillips, M. M., Suntzeff, N. B., Maza, J., González, L. E., Roth, M., Krisciunas, K., Morrell, N., Green, E. M., Persson, S. E., \& McCarthy, P. J. 2003, Nature, 424, 651

Hauschildt, P. H. \& Baron, E. 1999, J. Comp. Applied Math., 109, 41

Hauschildt, P. H., Baron, E., \& Allard, F. 1997a, ApJ, 483, 390

Hauschildt, P. H., Baron, E., Starrfield, S., \& Allard, F. 1996, ApJ, 462, 386

Hauschildt, P. H., Schwarz, G., Baron, E., Starrfield, S., Shore, S., \& Allard, F. 1997b, ApJ, 490, 803

Hillebrandt, W. \& Leibundgut, B., eds. 2003, ESO Astrophysics Symposia (Berlin: Springer)

Hillebrandt, W., Reinecke, M., \& Niemeyer, J. 2000, in Proceedings of Festschrift for Joe Monaghan, ed. J. Mould (Cambridge: Cambridge Univ. Press), astro-ph/0005341

Jordan, G. I., Fisher, R., Townsley, D., Calder, A., Graziani, C., Asida, S., Lamb, D., \& Truran, J. 2007, ArXiv Astrophysics e-prints

Kasen, D. \& Woosley, S. E. 2007, ApJ, 656, 661 
Khokhlov, A. 1991a, A\&A, 245, 114

—. 1991b, A\&A, 246, 383

-. 1991c, A\&A, 245, L25

Khokhlov, A. M. 1993, ApJ, 419, 200

Kirshner, R. P. et al. 1993, ApJ, 415, 589

Kozma, C., Fransson, C., Hillebrandt, W., Travaglio, C., Sollerman, J., Reinecke, M., Röpke, F., \& Spyromilio, J. 2005, A\&A, 437, 983

Lentz, E., Baron, E., Branch, D., \& Hauschildt, P. H. 2001, ApJ, 557, 266

Mazzali, P. A., Röpke, F. K., Benetti, S., \& Hillebrandt, W. 2007, Science, 315, 825

Nomoto, K., Thielemann, F.-K., \& Yokoi, K. 1984, ApJ, 286, 644

Nugent, P., Baron, E., Branch, D., Fisher, A., \& Hauschildt, P. 1997, ApJ, 485, 812

Nugent, P., Baron, E., Hauschildt, P., \& Branch, D. 1995a, ApJ, 441, L33

Nugent, P., Phillips, M., Baron, E., Branch, D., \& Hauschildt, P. 1995b, ApJ, 455, L147

Phillips, M. M. 1993, ApJ, 413, L105

Phillips, M. M., Lira, P., Suntzeff, N. B., Schommer, R. A., Hamuy, M., \& Maza, J. 1999, AJ, 118, 1766

Plewa, T., Calder, A. C., \& Lamb, D. Q. 2004, ApJ, 612, L37

Reinecke, M., Hillebrandt, W., \& Niemeyer, J. C. 1999a, A\&A, 347, 739

-. 2002a, A\&A, 386, 936

-. 2002b, A\&A, 391, 1167

Reinecke, M., Hillebrandt, W., Niemeyer, J. C., Klein, R., \& Gröbl, A. 1999b, A\&A, 347, 724

Reinecke, M., Niemeyer, J. C., \& Hillebrandt, W. 2002c, New Astronomy Review, 46, 481

Riess, A. G., Press, W. H., \& Kirshner, R. P. 1995, ApJ, 438, L17

-. 1996, ApJ, 473, 88 
Röpke, F. \& Hillebrandt, W. 2005a, A\&A, 431, 635

Röpke, F. K. 2005, A\&A, 432, 969

Röpke, F. K., Gieseler, M., Reinecke, M., Travaglio, C., \& Hillebrandt, W. 2006, A\&A, 453, 203

Röpke, F. K. \& Hillebrandt, W. 2005b, A\&A, 439, L29

Röpke, F. K., Hillebrandt, W., Niemeyer, J. C., \& Woosley, S. E. 2006, A\&A, 448, 1

Röpke, F. K., Woosley, S. E., \& Hillebrandt, W. 2007, ApJ, 660, 1344

Thomas, R. C. et al. 2007, ApJ, 654, L53 